\title{
The Influence of Motivational Interviewing on Patients With Inflammatory Bowel Disease: A Systematic Review of the Literature
}

\author{
Scott T. Wagonera, c, Jan Kavookjian ${ }^{\mathrm{b}}$
}

\begin{abstract}
Inflammatory bowel disease (IBD) is a condition accompanied by several physical and often psychological symptoms (e.g., depression). Treatments generally involve dietary modifications and prescription medications. Of concern, non-adherence rates with prescription medications for this population have been reported to be between $30 \%$ and $45 \%$. In order to examine an intervention that has shown promise in improving adherence, researchers systematically reviewed the literature in order to determine the impact of a motivational interviewing (MI) intervention on outcomes for individuals diagnosed with IBD. The outcomes assessed were broad and included, among others, the target behaviors of medication adherence and advice-seeking, and also patient-perceived provider empathy. Results suggest that MI can be effective in improving outcomes for individuals with IBD since patients experienced improved adherence rates, displayed greater advice-seeking behavior, and perceived providers as having more empathy and better communication skills. Further research is required since the pool of retained studies is small, evidencing a paucity of literature focusing on this evidencebased health behavior intervention for the behaviors needed to optimally manage IBD. Further, only adults were examined in these studies, so generalizations to children and adolescents are limited.
\end{abstract}

Keywords: Motivational interviewing; Inflammatory bowel disease; Adherence; Ulcerative colitis; Crohn's disease

\section{Introduction}

In the United States, approximately 1.3 million people are afflicted with inflammatory bowel disease (IBD) [1]. Comprised of two primary diagnoses, Crohn's disease and ulcerative colitis, IBD is marked by chronic inflammation of the gastrointes-

Manuscript submitted May 22, 2017, accepted May 30, 2017

aDepartment of Psychology, Auburn University, Auburn, AL, USA

bDepartment of Health Outcomes Research and Policy, Auburn University, Auburn, AL, USA

${ }^{\mathrm{c} C o r r e s p o n d i n g ~ A u t h o r: ~ S c o t t ~ T . ~ W a g o n e r, ~ D e p a r t m e n t ~ o f ~ P s y c h o l o g y, ~ A u b u r n ~}$ University, Auburn, AL 36849, USA. Email: swagoner@auburn.edu

doi: https://doi.org/10.14740/jocmr3081w tinal tract [2] and fluctuations of disease activity [3]. Individuals with IBD can experience a vast array of negative symptoms such as diarrhea, abdominal pain, rectal bleeding, and weight loss $[4,5]$. Complications of IBD extend beyond digestive issues and include fever, arthritis [6], and fatigue [7]. In addition to physical symptoms, individuals with IBD are known to experience more frequent, and clinically significant, internalizing symptoms (e.g., depression, anxiety) relative to healthy counterparts $[6,8]$.

IBD is managed through a variety of options which are implemented in order to achieve a remission of symptoms [9]. Common treatments include anti-inflammatory and immunosuppressive medications [10], and surgery is sometimes pursued [6]. Further, individuals with IBD are often required to make dietary adjustments and adhere to daily oral medication regimens to strive towards improved outcomes [1]. Though medication adherence comes with numerous health benefits, a review of adherence to oral medications concluded that nonadherence rates for patients with IBD fall mostly in the 30$45 \%$ range [11]. Additionally, non-adherence is particularly a concern for those experiencing a remission since not taking their medication(s) puts them at risk of returning to debilitating symptoms and poor outcomes [12].

Given the physical and psychosocial symptoms associated with IBD, in addition to concerning rates of non-adherence, it is important to consider behavioral interventions which may improve outcomes for those with IBD. One such intervention which may prove useful for helping IBD patients with behavior change decision-making is the patient-centered communication skills set and way of being known as motivational interviewing (MI). This way of being, known as the spirit of MI, involves providers communicating in supportive, caring, and empathic ways to resolve a patient's ambivalence for health behavior change [13].

In an MI intervention, a provider's communication style is critical in inspiring the patient's desires to change behavior [14]. Specifically, providers ask, listen, and inform their patients when attempting to elicit motivation to change [15]. Notably, this process includes the provider centering on the patient through implementing patient-centered communication principles and skills including expressing empathy, reflective listening [16], developing discrepancies between patient's current behaviors and goals, rolling with resistance, and supporting patient's self-efficacy to change health behaviors [17].

MI has shown promise in impacting numerous health be- 
haviors (e.g., diet, exercise, diabetes care, adherence) [18, 19] and general patient outcomes (e.g., mood) [20]. It would be useful to examine evidence and gaps in the literature for the utility and impact of MI interventions in addressing the complex behaviors required to manage IBD.

To date, no known review has been conducted to describe the evidence base for MI interventions and outcomes in patients with IBD. Given the complex nature of IBD and the importance of adherence to medication(s) to increase the probability of improved outcomes, this is an important literature gap to fill. As established previously, MI is successful in improving adherence as well as certain psychosocial outcomes in other populations, target behaviors, and conditions; therefore, since MI has the potential to improve outcomes for those who need to make health behavior change decisions, this review systematically explores and reports evidence and gaps in the literature for the utility and impact of MI on behavior changes required of patients with IBD and other outcomes.

The objective of this review is to use a modified Cochrane method for systematic search and review of the literature and determine: 1) the extent to which MI impacts outcomes for those diagnosed with IBD, and 2) optimal MI methods used to achieve desired outcomes. The scope of this review will address implications for practice and for research since no known reviews of MI interventions have been conducted for IBD management.

All English-language articles published in peer-reviewed journals during the 1990 through May 2017 timeframe were considered. To be included, studies had to: 1) include a sample of persons of any age diagnosed with IBD, 2) include and describe a form of MI as an intervention, and 3) assess for at least one target behavior change and outcome variable (e.g., adherence, disease severity, internalizing symptoms, quality of life) studied in association with the MI intervention.

\section{Materials and Methods}

\section{Search strategy}

A systematic review was conducted utilizing a modified Cochrane approach. Databases in psychology (PsycINFO), nursing (CINAHL), pharmacy (International Pharmaceutical Abstracts), and medicine (Medline) were searched, as these databases were likely to provide the most results related to individuals with IBD. Additionally, manual searches of reference lists within target articles were also conducted. Search terms included: motivational interviewing, multi-component, inflammatory bowel disease, IBD, gastroenterology, gastrointestinal, Crohn's disease, and ulcerative colitis. Lastly, studies were excluded if they: 1) were a review/commentary, 2) did not present original data, or 3) did not include either a control group or baseline measurement with which to compare an outcome to.

\section{Data extraction and analysis}

Information about study methodology, participant charac- teristics (e.g., age, diagnosis, treatment regimen), interventions, and outcomes were extracted from the articles and compiled in a data form for analysis. All articles retained for the review were evaluated for study quality in order to report claims for validity and generalizability as well as included methods increasing the likelihood of preventing bias. In order to evaluate risk of bias, the Cochrane methodological quality rating system was used to evaluate six sources of bias; these included sequence generation, allocation concealment, blinding, incomplete outcome data, selective outcome reporting, and other potential threats to validity. In this system, articles are rated across the six bias sources by either "yes" (low risk of bias), "no" (high risk of bias), or "unclear" risk of bias.

\section{Results}

Fifty-two articles were identified in the initial database search, and three were obtained through manual searching among references lists. Figure 1 shows the trial flow diagram of the search and screening tiers used in the review process.

Following the removal of duplicates $(n=7)$, exclusions were made based on brief-title and abstracting screenings ( $\mathrm{n}$ =33). The remaining 15 articles were given a full text review. At the conclusion of the full text review process, four articles were deemed appropriate for retention. Reasons for exclusion included: one report of ongoing work, one examining barriers to colonoscopy, four with a non-IBD population, one editorial, one review, one with a therapy that did not include MI, and one case study.

\section{Study and intervention types, sample descriptions, and outcomes assessed}

As can be seen in Table 1 [21-24] of description of retained studies, two of the four retained studies were randomized controlled trials (RCTs). The remaining studies were quasi-experimental designs wherein participants were retrospectively compared to themselves, a general population baseline, or their pre- and post-ratings were compared to determine differences.

MI intervention characteristics were heterogeneous and included one-on-one meetings with a physician $(n=2)$, a telephone intervention implemented by a registered nurse ( $\mathrm{n}$ $=1$ ), and an MI session implemented as the first of a sixsession mindfulness-based intervention given by a Master'slevel therapist $(n=1)$. Intervention lengths were also varied and included: telephone calls over a 6-month period averaging $13 \mathrm{~min}$ each, one 45-min routine consultation, one 40min MI session followed by five 40-min mindfulness-based sessions occurring over 16 weeks, and one 20- to 30-min MI session.

Not all studies detailed the training of interventionists [21, 22]. Of the two studies that did, one described the interventionist as experienced and qualified in counseling and psychotherapy [23]. The other study described the training as including 


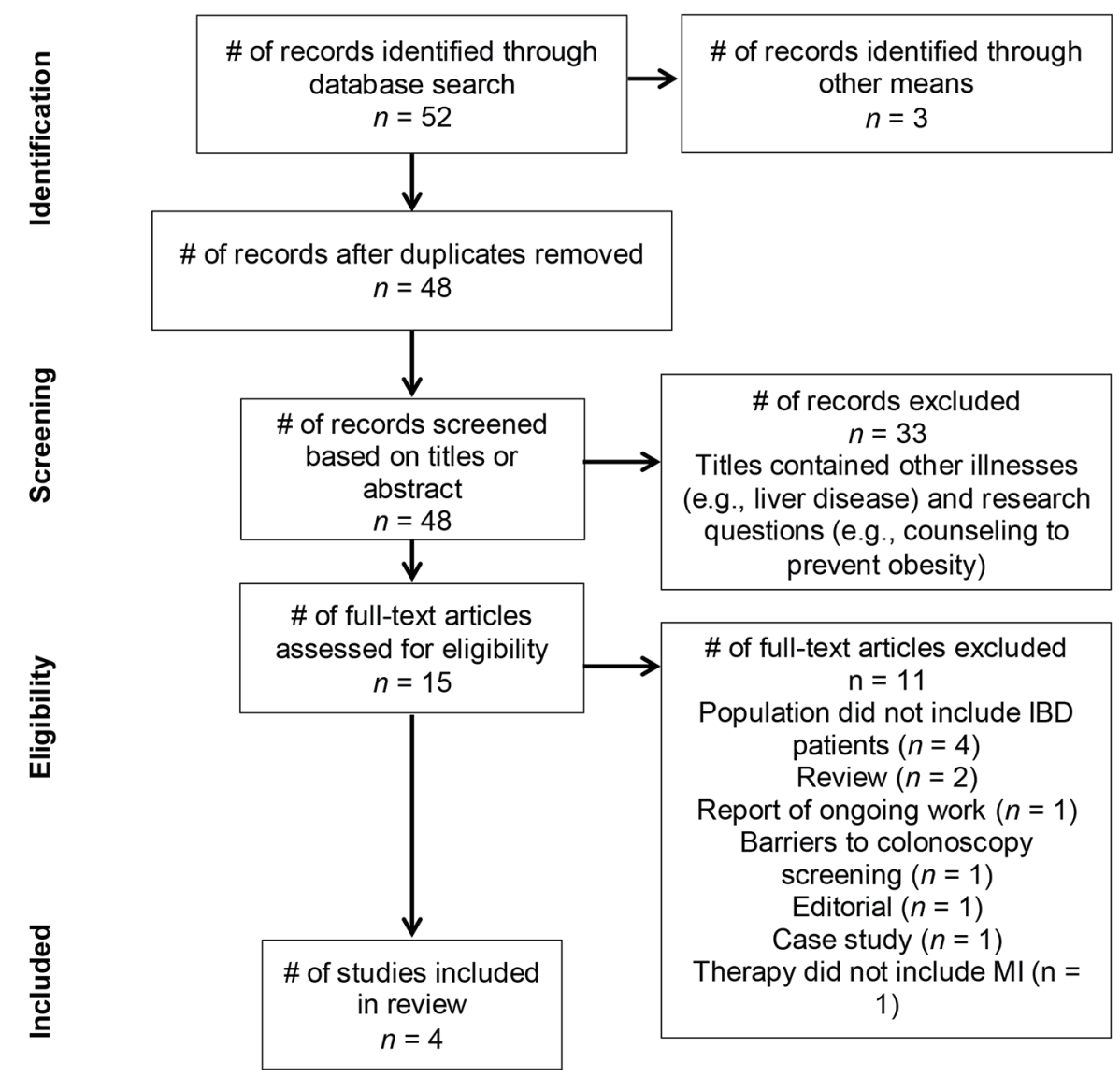

Figure 1. PRISMA flow diagram detailing trial search and review tiers.

an 8-h program on the study's specific counseling intervention coupled with individual supervision and weekly team meetings with research personnel [24].

Of the four retained studies, two were comprised solely of patients diagnosed with ulcerative colitis. Sample sizes ranged from 45 to 278 patients, and patients across studies ranged in age from 20 to 82 years-old. Outcomes measured were varied and included, among others, adherence, patient satisfaction with provider, quality of life, and patient-perceived provider empathy.

\section{Adherence}

Adherence was the most commonly assessed outcome, examined by three of the four studies $[21,22,24]$. Though adherence was generally obtained through self-report, one study assessed adherence through patient urine samples [22]. Across studies, an MI intervention was related to positive outcomes in adherence. For example, patients who were exposed to an intervention that incorporated a one-on-one session had significantly higher adherence rates than a control group [22]. Relatedly, Cook and colleagues [24] found that patients exposed to a telephone-based intervention had higher rates of self-reported adherence than a comparison population base- line. Lastly, Mocciaro and colleagues [21] reported that patients who received MI as part of a routine consultation were $95.6 \%$ adherent to their medications at a follow-up visit based on their self-reports.

\section{Quality of life}

One study assessed quality of life [23], and though the intervention group as a whole did not experience a significantly greater quality of life than the control group, the intervention subgroup with irritable bowel syndrome symptoms did experience significantly greater quality of life post-intervention compared to their own baseline.

\section{Provider aspects}

One study examined patients' perceptions of their providers following the patient-centeredness of an MI intervention by examining perceptions of empathy and satisfaction with the encounter(s) [21]. Patients reported their providers as being significantly more empathic and skilled at communication than previous providers. In addition, patients reported being significantly more satisfied with their provider compared to previous providers. 


\begin{tabular}{|c|c|c|c|c|}
\hline 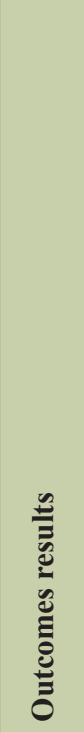 & 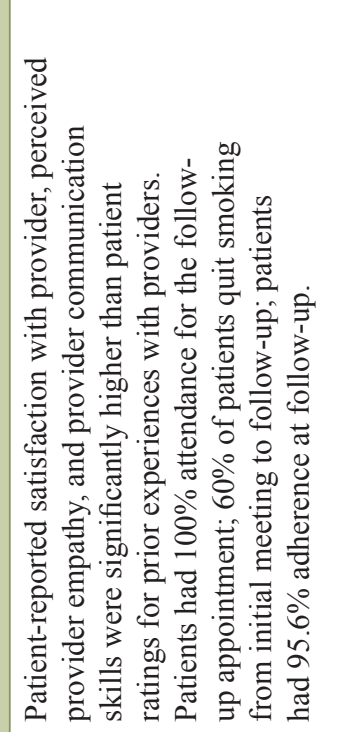 & 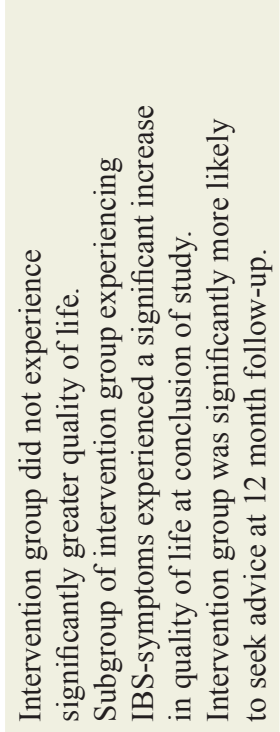 & 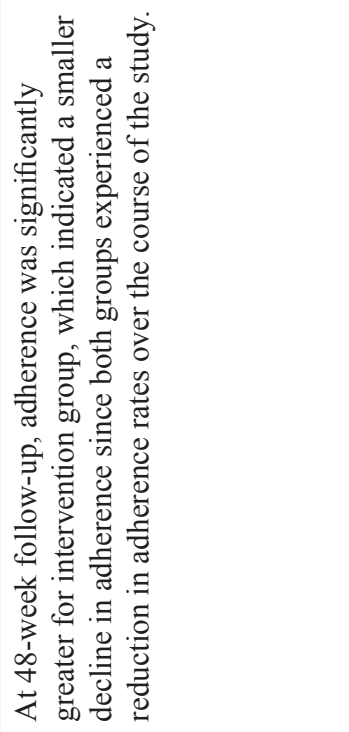 & 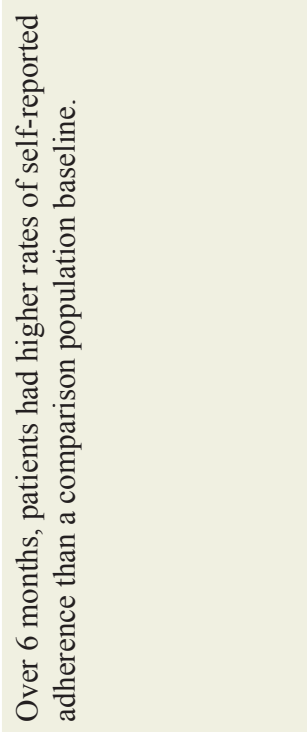 \\
\hline 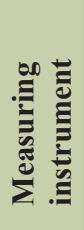 & 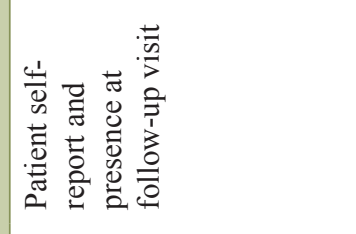 & 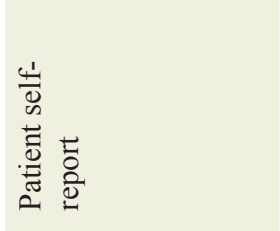 & 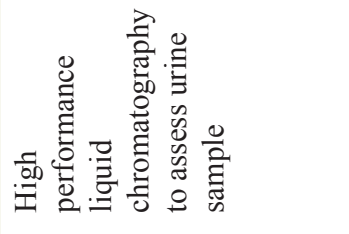 & 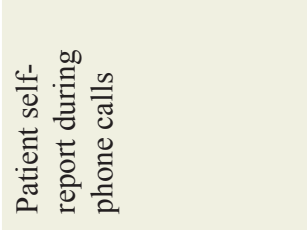 \\
\hline 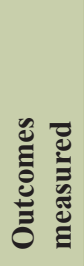 & 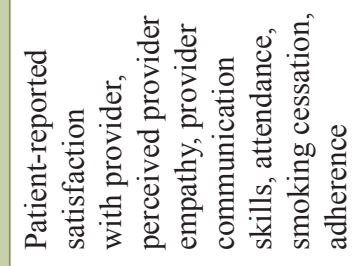 & 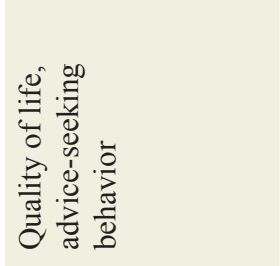 & 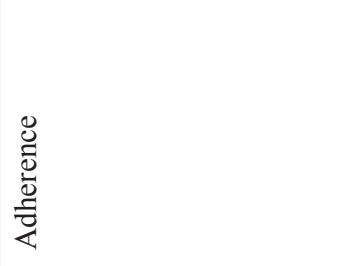 & 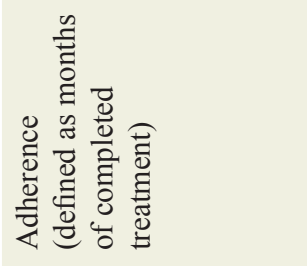 \\
\hline 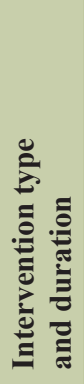 & 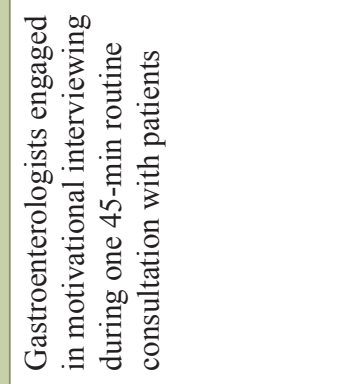 & 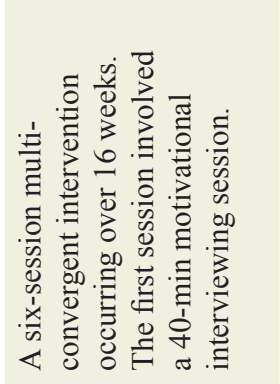 & 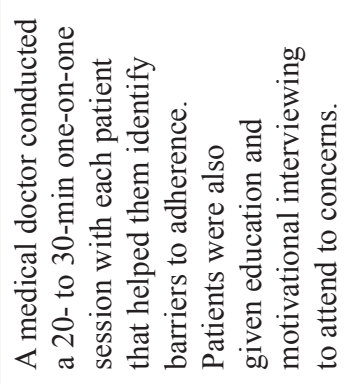 & 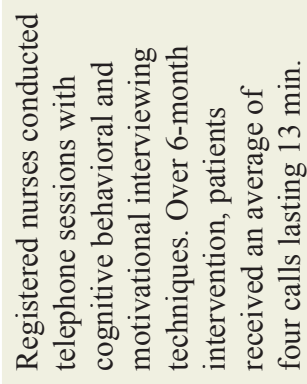 \\
\hline 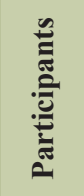 & 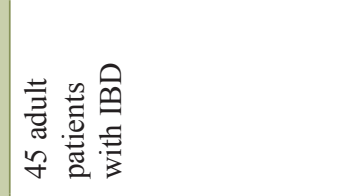 & 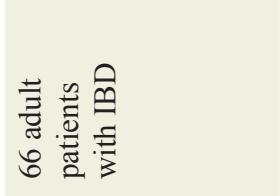 & 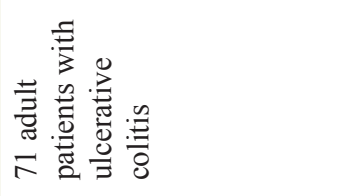 & 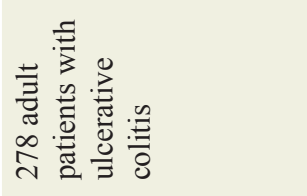 \\
\hline 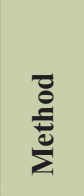 & 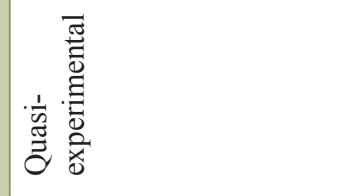 & 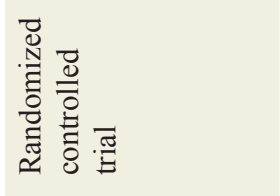 & 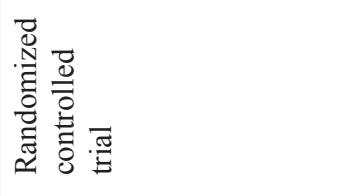 & 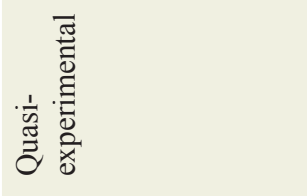 \\
\hline 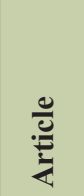 & 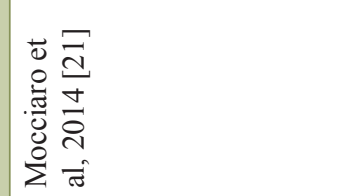 & 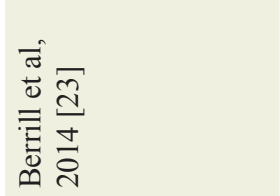 & 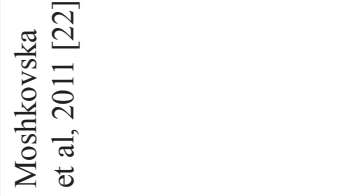 & 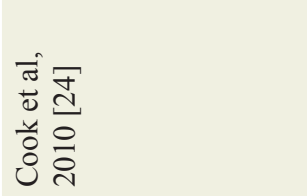 \\
\hline
\end{tabular}




\section{Miscellaneous outcomes}

As mentioned previously, the retained articles examined numerous outcomes, and results suggest that MI interventions were effective at improving these outcomes. For example, patients who received an MI intervention were significantly more likely to seek advice than those in a control group [23]. Further, results from Mocciaro and colleagues [21] suggested that high attendance for follow-up appointments and smoking cessation were positive outcomes related to an MI intervention.

\section{Evaluation of methodological quality}

Table 2 shows a complete description of methodological quality assessment. Two of the retained studies were quasi-experimental designs, and, as such, there are inherent risks of bias as noted in the table. Further, there is the potential for risk in one of the RCTs [23] given that the randomization procedures are not well described. Studies were mixed on whether interventionists knew if patients were in a control group $(n=2)$ or were blind $(n=2)$. Relatedly, the majority of studies $(n=3)$ specifically stated patients knew they were in an intervention group; one study was unclear about patient blinding.

\section{Discussion}

\section{Main results}

The systematic approach applied in this review identified only a small body of literature approaching the application of an evidence-based, patient-centered behavior change intervention like MI in patients with IBD, for whom the disease demands a lot of self-management changes, including medication adherence. Across studies, being exposed to an MI intervention appears to have positively benefitted patients participating in the retained studies. Regarding the most studied outcome, adherence, results suggest that MI was effective at both improving and sustaining adherence. For other outcomes (e.g., perceived provider patient-centeredness, quality of life), MI also appears to have been an effective intervention; though, regarding quality of life, only a specific subset (i.e., those experiencing certain symptoms) noticed an improvement in quality of life. Positively, these effects are related to interventions of varying type (e.g., telephone, in-person) and duration (e.g., one session, 6 months).

\section{Implications for practice}

These results are a strong preliminary investigation into the use of MI for individuals diagnosed with IBD. Many important issues for IBD were detailed (e.g., adherence, quality of life); however, other important issues related to IBD (e.g., internalizing concerns) appear to be absent from the literature. Approaching patients suffering with IBD in a patient-centered, autonomy-supported way can help facilitate their decisionmaking to engage in self-management behaviors that will help improve symptoms, increase the likelihood of remission, and likely improve quality of life. For example, providers can be collaborative and use incremental goal setting to help patients feel optimistic about making health behavior changes (e.g., improve adherence). Further, using agenda setting (i.e., allowing patients to decide what topics to focus on) and permission asking before giving advice may help patients feel respected and empowered to take active roles in their care.

\section{Implications for research}

Studies retained in this review exhibited heterogeneous designs and methods for implementation and measurement, making it impossible to identify a "best practice" evidence for implementation of MI in IBD patient populations. In addition, it is unclear whether the varied interventionists were fully and specifically trained in MI since most studies did not specify what the training included, nor did they report assessing intervention fidelity to MI in the actual patient encounters. These are important study methods to assess when making claims for validity of a complex behavioral intervention strategy set like MI. In addition, the smaller samples sizes, varied ways of measuring adherence, missing patient characteristics that could have been associated with outcomes (e.g., severity of disease), and varied number and duration of MI encounters within these study designs create variability that contributes to the suggestion that further research is needed, particularly when a systematic search and review produced only four articles for retention.

Research should be conducted to expand upon this work simply to provide more evidence on the effectiveness of MI on various IBD outcomes. Specifically, future work can investigate the effectiveness of MI for improving internalizing symptoms. Additionally, research can investigate the extent to which MI differentially impacts those who are in remission and those who are experiencing a flare of symptoms. Further, the current research does not approach the question of effectiveness of MI for children and adolescents. This is a critical gap to fill since approximately $25 \%$ of IBD cases present prior to age 20 [25].

\section{Strengths and limitations}

To our knowledge, this was the first known study to review the literature on the association between MI and outcomes for individuals diagnosed with IBD. This study utilized a modified Cochrane approach for search and review of the literature. Though this was the first known review approaching this set of research questions, this review was able to detail many IBD outcomes associated with an MI intervention.

Despite the strengths of this review, there are limitations which must be discussed. First, not every article retained was an RCT; therefore, drawing definite conclusions about the effectiveness of MI is difficult for some of the retained articles. 


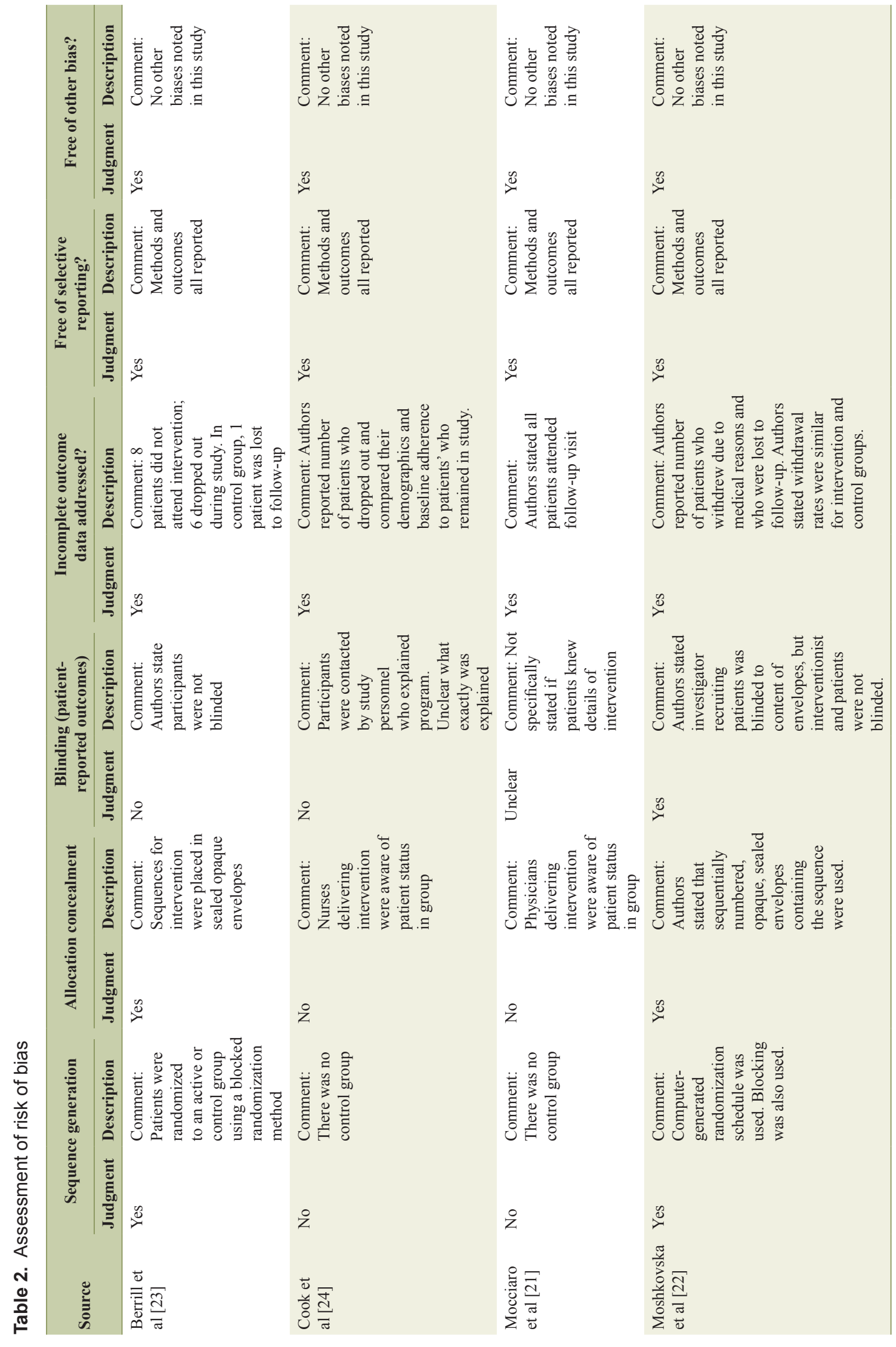


Further, the number of articles included is relatively small. Because this appears to be an emerging area of research, the results presented should be considered under the notion that future work is needed to form more concrete conclusions.

\section{Conclusions}

Promisingly, initial results suggest patients with IBD respond positively to the implementation of MI. Providers can consider MI when they want to improve relationships with patients and be perceived as more empathic and skilled at communication. Further, providers may also find MI as an appropriate approach in helping patients adhere to their medications. Given the emerging nature of this research, providers should consider a patient-centered approach like MI in helping facilitate patient decision-making for behavior changes needed to manage IBD. Additional research is warranted and should be expanded to explore the effectiveness of MI for children and adolescents with IBD given the prevalence rates for those groups.

\section{Conflicts of Interest and Sources of Funding}

Jan Kavookjian reports being faculty for the Merck Speakers Bureau for non-branded medical education for the topic, Relationship Centered Care (Motivational Interviewing). For Scott Wagoner, none were declared.

\section{References}

1. Kappelman MD, Rifas-Shiman SL, Kleinman K, Ollendorf D, Bousvaros A, Grand RJ, Finkelstein JA. The prevalence and geographic distribution of Crohn's disease and ulcerative colitis in the United States. Clin Gastroenterol Hepatol. 2007;5(12):1424-1429.

2. Fiocchi C. Inflammatory bowel disease: etiology and pathogenesis. Gastroenterology. 1998;115(1):182-205.

3. Dubinsky M. Special issues in pediatric inflammatory bowel disease. World J Gastroenterol. 2008;14(3):413420.

4. Auvin S, Molinie F, Gower-Rousseau C, Brazier F, Merle V, Grandbastien B, Marti R, et al. Incidence, clinical presentation and location at diagnosis of pediatric inflammatory bowel disease: a prospective population-based study in northern France (1988-1999). J Pediatr Gastroenterol Nutr. 2005;41(1):49-55.

5. Sawczenko A, Sandhu BK. Presenting features of inflammatory bowel disease in Great Britain and Ireland. Arch Dis Child. 2003;88(11):995-1000.

6. Greenley RN, Hommel KA, Nebel J, Raboin T, Li SH, Simpson P, Mackner L. A meta-analytic review of the psychosocial adjustment of youth with inflammatory bowel disease. J Pediatr Psychol. 2010;35(8):857-869.

7. van Langenberg DR, Gibson PR. Systematic review: fatigue in inflammatory bowel disease. Aliment Pharmacol Ther. 2010;32(2):131-143.

8. Mackner LM, Crandall WV. Brief report: psychosocial adjustment in adolescents with inflammatory bowel disease. J Pediatr Psychol. 2006;31(3):281-285.

9. Grisham MB. Oxidants and free radicals in inflammatory bowel disease. Lancet. 1994;344(8926):859-861.

10. Pithadia AB, Jain S. Treatment of inflammatory bowel disease (IBD). Pharmacol Rep. 2011;63(3):629-642.

11. Jackson CA, Clatworthy J, Robinson A, Horne R. Factors associated with non-adherence to oral medication for inflammatory bowel disease: a systematic review. Am J Gastroenterol. 2010;105(3):525-539.

12. Lakatos PL. Prevalence, predictors, and clinical consequences of medical adherence in IBD: how to improve it? World J Gastroenterol. 2009;15(34):4234-4239.

13. Levensky ER, Forcehimes A, O'Donohue WT, Beitz K. Motivational interviewing: an evidence-based approach to counseling helps patients follow treatment recommendations. Am J Nurs. 2007;107(10):50-58; quiz 58-59.

14. Miller WR, Rollnick S. Motivational Interviewing: Helping People Change, 3rd Edition. New York, NY, US: Guilford Press; 2013. http://spot.lib.auburn.edu/login?url=http://search.ebscohost.com/ login.aspx?direct $=$ true $\& \mathrm{db}=$ psyh $\& A N=2012-17300$ 000\&site $=$ ehost-live.

15. Rollnick S, Miller WR, Butler CC. Motivational Interviewing in Health Care: Helping Patients Change Behavior. New York, NY, US: Guilford Press; 2008. http://spot. lib.auburn.edu/login?url=http://search.ebscohost.com/ login.aspx?direct $=$ true $\& \mathrm{db}=$ psyh $\& A N=2008-01398$ 000\&site $=$ ehost-live.

16. Gillaspy SR, Litzenburg CC, Leffingwell TR, Miller MB. Training in motivational interviewing as a best training practice in pediatric psychology: relationship to core competencies. Clin Pract Pediatr Psychol. 2015;3(3):225232.

17. Miller WR, Rollnick S. Motivational Interviewing: Preparing People to Change Addictive Behavior. New York, NY, US: Guilford Press; 1991. http://spot.lib. auburn.edu/login?url=http://search.ebscohost.com/ login.aspx?direct $=$ true $\& \mathrm{db}=$ psyh $\& A N=1991-98398$ 000\&site $=$ ehost-live.

18. Martins RK, McNeil DW. Review of Motivational Interviewing in promoting health behaviors. Clin Psychol Rev. 2009;29(4):283-293.

19. Ogedegbe G, Chaplin W, Schoenthaler A, Statman D, Berger D, Richardson T, Phillips E, et al. A practicebased trial of motivational interviewing and adherence in hypertensive African Americans. Am J Hypertens. 2008;21(10):1137-1143.

20. Watkins CL, Wathan JV, Leathley MJ, Auton MF, Deans CF, Dickinson HA, Jack CI, et al. The 12-month effects of early motivational interviewing after acute stroke: a randomized controlled trial. Stroke. 2011;42(7):1956-1961.

21. Mocciaro F, Di Mitri R, Russo G, Leone S, Quercia V. Motivational interviewing in inflammatory bowel disease patients: a useful tool for outpatient counselling. Dig Liver Dis. 2014;46(10):893-897.

22. Moshkovska T, Stone MA, Smith RM, Bankart J, Baker R, Mayberry JF. Impact of a tailored patient preference intervention in adherence to 5-aminosalicylic acid 
medication in ulcerative colitis: results from an exploratory randomized controlled trial. Inflamm Bowel Dis. 2011;17(9):1874-1881.

23. Berrill JW, Sadlier M, Hood K, Green JT. Mindfulnessbased therapy for inflammatory bowel disease patients with functional abdominal symptoms or high perceived stress levels. J Crohns Colitis. 2014;8(9):945-955.
24. Cook PF, Emiliozzi S, El-Hajj D, McCabe MM. Telephone nurse counseling for medication adherence in ulcerative colitis: a preliminary study. Patient Educ Couns. 2010;81(2):182-186.

25. Kelsen J, Baldassano RN. Inflammatory bowel disease: the difference between children and adults. Inflamm Bowel Dis. 2008;14(Suppl 2):S9-11. 